\title{
Scaling For SETI: All The Sky, All The Time
}

\author{
Kent Cullers \\ SETI Institute, 2035 Landings Drive, Mountain View, CA 94043 USA
}

\begin{abstract}
The Search for Extraterrestrial Intelligence (SETI) has been, in its modern sense, a search for signs of technology beyond the Solar System. Such a search is a natural companion to visual and radio astronomy, which searches the electromagnetic spectrum to glean information about natural cosmic events. To succeed, SETI must demonstrate the existence of signals that cannot be explained by natural events and that are not of terrestrial origin.
\end{abstract}

\section{Introduction}

Over the last forty years, SETI has concentrated on the microwave portion of the electromagnetic spectrum. First, this is because the signature of terrestrial technology is so different from that of the Sun in this band. Second, using radio technology, this band falls at a cosmic noise minimum, thus enhancing the signal/noise ratio of any putative extraterrestrial transmission.

The present-day Earth emits strong radio, TV, and radar signals. These contain large amounts of power that are concentrated either in very narrow regions of frequency or short segments of time. Such signals are generally quite different from natural electromagnetic events, which are significantly less concentrated. Digital spectrometers easily slice incoming data into segments which may, perhaps, contain an extraterrestrial signal with characteristics similar to those of our technology while containing only small amounts of the more spread out natural signals and noise. Such SETI analysis yields data windows in which some of our Earth-based signals are a million times brighter than the electromagnetic radiation from our Sun. Generally, it is the concentrated nature of technology, modeled on Earth, for which SETI combs the distant stars.

The Cyclops Study (Oliver \& Billingham 1972) demonstrated that technology of that era could detect a "twin" of the Earth. That is, antenna arrays and analogue processing of that time could sequentially search a million stars and find beacons radiating a Gigawatt of power isotropically into space. Although no terrestrial transmitters do this exactly, such a transmitter was certainly possible using 1970s technology. In the same way, arrays with sufficient collecting area did not exist at that time, but they could be built using analogue techniques to form a single beam with bandwidth wide enough to process data from several hundred megahertz of spectrum.

This demonstrated ability to detect a near twin of our rather primitive technology, sparked a host of SETI experiments. Recently, the SETI Institute has published a study of directions for SETI, considering the evolution of technology 
since 1970. It examines how to search for not only a twin of our present technology but how one might extrapolate current technological trends and search for modestly more advanced analogues of Earth.

The primary difference between Cyclops and the SETI Institute study conducted a quarter century later (Ekers et al. 2002), is the phenomenal growth in the power of computers. Both beam formation and data analysis can be performed digitally today, providing more capability at lower cost. Such new processing allows simultaneous coverage of many targets over wide ranges of frequency. Time reversing these same capabilities can, in principle, produce powerful beacons that beam stars of interest. Arrays, forming not one analogue beam, but multiple digitally synthesized beams can combine data to remove one of the growing problems of the last quarter century, terrestrial interference. New sensors and electronics, using standard telescopes, now perform searches for highly directional, short laser pulses which may momentarily be bright even against a star at optical wavelengths. Though our technology does not do this presently, it is certainly within the capability of our near "twin". Computing power is growing dramatically, doubling about every eighteen months.

Such cheap computing, eventually synthesizing quite large arrays from small, inexpensive elements, will allow many beams to simultaneously cover the sky. This condition of modern technological progress led to the title of this talk and a goal of a new SETI vision, "All The Sky, All The Time" over a wide range of frequencies of interest from microwave to optical. Further, it led to the expectation that the power of searches may grow geometrically instead of linearly, permitting significant searches of the Galaxy within a human lifetime.

\section{Principals of Antenna Synthesis}

The following is a general tutorial on the SETI proposals for the next two decades. It describes the types of systems considered, rather than systems actually existing today. In essence, it considers concepts and possibilities with only passing reference to some of the actual projects already under construction.

It is possible to use many analogies to explain how computers do signal detection with sensitivities beyond that of humans. As a blind physicist, I often use audio analogies to guide my intuition in developing signal processing algorithms.

When listening to audio signals, our ears naturally convey spectral knowledge. Basically, we recognize the regularity of pulsed signals like those from a ticking clock and the dominance of certain frequencies as when middle $\mathrm{C}$ is played on a violin. Since it is possible to shift electromagnetic signals from very high to low frequencies and convert them to sound, it is useful to think of radio signals in this intuitive way. When humans analyze signals, we may listen to them or view them on a screen. In either case, compared to what computers can do today, signals must be enhanced in their characteristics for human senses to perceive them.

Using standard computational methods such as the Discrete Fourier Transform, it is easy to synthesize filters with bandwidths of a hundredth of a Hertz. Individual channels of this kind contain very little cosmic noise but may well contain almost all the power in the carrier of a stable TV or radio signal. Car- 
riers are pure sinusoidal components that accompany the normal programming information of a signal. These allow receivers to be built at low cost. Thus, power is spent in the carrier of a transmitter so that millions of receivers can use simple circuitry to perform signal decoding. The carrier is filtered out of the data stream in this decoding operation.

If a signal is shifted directly in to an analysis band, the carrier is an outstanding point of power density. Tuning across bands of such shifted signals, one perceives the whistles of pure carriers rather than the programming information of a transmission.

A carrier signal can sweep across hundreds of narrow channels before the human ear can perceive a frequency change. Thus, the signal/noise heard by the human ear is degraded by perhaps a factor of a thousand compared to that in a narrow synthesized channel.

Of course, signals stable at the point of transmission, say an alien planet, will not be stable when received on the Earth. Such a planet, like the Earth itself, is rotating and orbiting. The alien signal is in accelerated motion relative to the terrestrial receiver, and the Doppler effect causes the received frequency to drift as the relative velocity changes. This same effect accounts for the change in pitch of a train whistle as the train passes an observer along a track.

In order to detect a signal carrier of this kind, one must sum the power along all tracks characterized by stable signals originating from rotating planets of terrestrial type. Though in general, drift rates due to rotation are sinusoidal, such sinusoidal behavior can be approximated by a matching linear segment during an observation. Thus, summing along signal tracks in a frequency-time plot is equivalent to summing from all possible starting channels along lines that differ in angle, depending on the acceleration. This is much more difficult than detecting a constant frequency signal fixed to the stars, requiring hundreds of times the computation of detecting a stable signal. However, since we wish to detect signals that are stable in the alien planets reference frame, we compute the power along drifting signal tracks.

In the same way we also detect pulsing sinusoidal signals, those which might be delivered by a radar systematically sweeping a distant sky. Such signals will deliver along the same drifting signal tracks as for carriers, trains of regularly spaced pulses. These are strong when the beam is on the Earth, and a simple thresholding technique can separate signal from noise. Such pulses can be seen with good sensitivity only if the length of the spectrometer window matches the length of the signal pulse. Thus, many signal analysis computations are necessary to detect pulses with wide ranges of length.

The regularity criterion is applied to maintain a low false alarm rate against the occurrence of randomly scattered noise fluctuations.

It may even be possible in the near future to detect strong transient events. These are more difficult than the discovery of a constant sinusoid or a regularly spaced pulse train for which relatively large amounts of time are spent to prove the existence of a signal. Transient signals will be detectable if the signal is strong, and multiple antennas cover the sky with a complete pattern of beams over wide frequency ranges. Such transients will be credible, if they are discovered in data from widely separated sites. This eliminates the possibility of earth-based interference or systematic error. It is worth noting that some of 
our strongest terrestrial signals are of this transient nature. Covering all the sky all the time requires formation of many beams with small elements, as will be explained below. Thus, though computing power will become inexpensive, collecting areas of antennas like the Omnidirectional Sky Survey (OSS) will be small at first, and signals, to be detectable, will have to be strong.

The new power of computing allows detection of transient signals, processing for new signal types and the elimination of terrestrial interference by subtraction or identification in databases.

Imagine this process in the audio domain where our intuition about signal processing is powerful. We hear echoes in a room with a sound system because the direct signal is also reflected to our ears from several surfaces. Echoes are the delayed and undistorted versions of the signal. We hear echoes when the elements that reflect signal are not positioned to produce coherent addition. Clearly, if we have the ability to add all the versions of the signal together with proper synchronization, the result is coherent. This can be done by situating the reflecting surfaces to perfectly focus the sound on a particular listener.

On the other hand, one can distribute receiving elements in a very arbitrary manner, record the signals from the individual elements, and synchronize the data streams in a computer to form a coherent output. If the distribution of antenna elements is especially fortuitous, say that of a parabola, recording at the elements is not necessary. All the signals arriving along the axis pass through the focus and are added together coherently. The difficulty is that construction of large accurate reflecting surfaces like parabolas is expensive. As the cost of computing drops, it is cheaper to build large collecting areas that gather the signals from many smaller elements, and use data processing to coherently add them. An advantage of this approach is the ability to sum the individual signals in many different ways, each sum synthesizing a large antenna pointed in a different direction.

In principle, antenna components can be used that are smaller than one wavelength in extent, each sensitive to most of the sky. This is because each element does not intercept a complete wavelength simultaneously, regardless of orientation, so cancellation across the element does not occur. Thus, one can synthesize beams over the whole sky, as for the Omnidirectional Sky Survey (OSS), using as many elements as computation allows. Consider the factors contributing to the beam computation if elements are placed in an arbitrary pattern. Basically, the computation is proportional to the product of the number of beams, the data bandwidth, and the number of elements used to form the beam. As elements become large, they see only wave fronts from a particular direction. Even small changes in orientation of the incoming signal causes cancellation across the antenna surface. Thus, areas composed of large elements require less computation, but all elements must be focused on a small part of the sky for the entire array to be utilized, and fewer beams can be formed. Small elements see much more of the sky, but many beams must be formed each using all the many elements. Thus, the beam forming computation is demanding for small elements, even when the total area being summed is relatively modest. The data processing per beam is also proportional to the bandwidth processed. The constant of proportionality is related to the complexity of the signal detec- 
tion algorithm. Generally, the beam forming computation tends to dominate the computational load when large antennas are formed with many small elements.

\section{The Allen Telescope Array (ATA)}

The ATA is now being designed to use the power of computers to form antennas. Its $3506 \mathrm{~m}$ diameter elements can be added in different ways to form the equivalent beam of a $100 \mathrm{~m}$ diameter dish pointing in several directions simultaneously. In addition, directions in the sky can be cause to have particularly low sensitivities so that satellites and other interfering sources can be rejected.

We can speed up the search by looking in many directions in the sky simultaneously over wider bandwidths and observing 24 hours per day. Spot beams can be formed to obtain faithful copies of satellite signals that can be subtracted from the data stream, canceling out interference. Over the next twenty years, we will take our search from a thousand stars to perhaps a hundred thousand stars through the power of computing.

During this same time period, we may scale up concepts like the ATA to form a Square Kilometer Array. This concept as it scales with available computing power, will support many beams and give the sensitivity necessary for new SETI and general radio astronomy. This ambitious scaling of present concepts will require a worldwide effort in radio astronomy. Although the ATA is a viable concept for this final antenna, it is only one of several ideas being tested at present.

\section{Conclusions}

If we wish to view all the sky all the time with small antennas of many elements using the OSS, view the sky in the optical, and have high sensitivity for hundreds of thousands of stars using the ATA, we need computing power and financial support. Examination of Fig. 1 shows some of the great computational needs.

Notice that the growth of the multiple beam computation is very large as well as that for signal processing for exotic signals.

Certainly when one considers the OSS, covering all the sky with many inexpensive elements, one can imagine the power of arrays growing geometrically as computing costs drop. For antennas of larger elements, such as the ATA, growth curves are more complex since the element cost is large. The total costs for the ATA OSS and optical searches are significant. They amount to an average cost of about $\$ 8,000,000$ per year. We could attempt to finance such projects on a flat budget.

On the other hand, for the OSS, an already exponentially growing system, it would be elegant, as well as ambitious, to allow the budget to also grow exponentially by a third per year as the power of its computers double every eighteen months. This implies a cost of tens of thousands per year at present, reaching by the end of the next two decades as much as $\$ 5,000,000$ per year. The combination of this aggressive budget and computing increases implies a doubling in search power of this project each year. With the aid of Moore's 


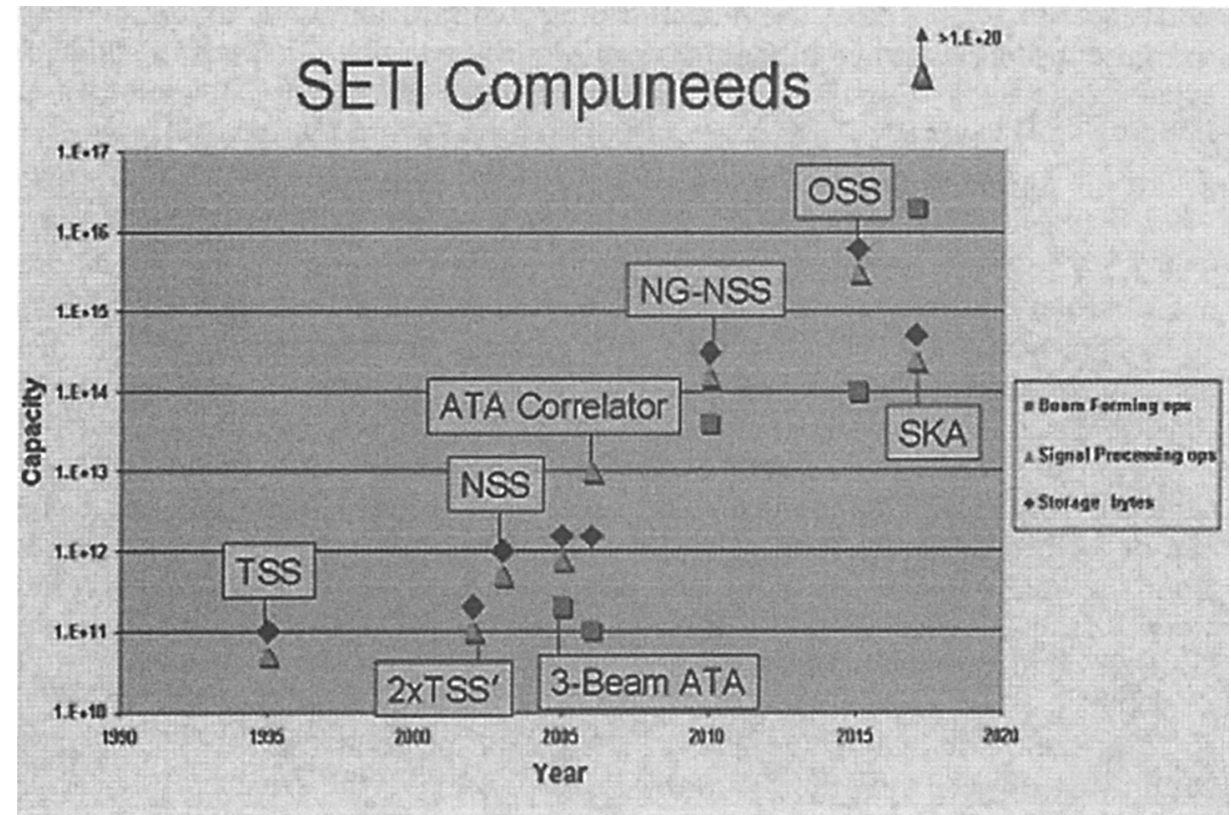

Figure 1. SETI Compuneeds

law, and if resources are available, the OSS can search a significant part of the Galaxy for strong, transient signals in the next two decades.

Acknowledgments. I wish to thank the SETI Institute for funding this work and the members of the SETI Science and Technology Working Group for so many grand ideas.

\section{References}

Oliver, B. M. \& Billingham, J. 1972, Project CYCLOPS: A Design Study of a System for Detecting Extraterrestrial Intelligent Life. Stanford UniversityAmes Research Center Summer Faculty Fellowship Program in Engineering Systems Design. June-August, 1971. NASA Contractor Report CR114445. Reprinted in 1996 by the SETI League and the SETI Institute

Ekers, R. D., Cullers, D. K., Billingham, J., \& Scheffer, L.K. 2002, in SETI 2020: A Roadmap for the Search for Extraterrestrial Intelligence, ed. R. D. Ekers, D. K. Cullers, J. Billingham, \& L. K. Scheffer, (Mountain View: SETI Press), 233 